\title{
An interactive online IT tool to aim the environmental surveillance of veterinary antibiotics in agriculture and pasture lands
}

\author{
Antonio Rodríguez $z^{\ddagger}$, Ana de la Torre ${ }^{\ddagger}$ \\ ‡INIA-CISA, Valdeolmos, Spain
}

Corresponding author: Antonio Rodríguez (antonio.rodriguez@inia.es)

Received: 18 May 2021 | Published: 28 May 2021

Citation: Rodríguez A, de la Torre A (2021) An interactive online IT tool to aim the environmental surveillance of veterinary antibiotics in agriculture and pasture lands. ARPHA Conference Abstracts 4: e68820. https://doi.org/10.3897/aca.4.e68820

\begin{abstract}
The undermining of the therapeutic effectiveness of antibiotics by their widespread use is causing the emergence of antimicrobial resistance, which is a major threat for both animal and human health. Since most veterinary antibiotics employed in livestock production are excreted essentially unaltered, they have been identified as major contributors of environmental contamination. However, the efforts of monitoring antimicrobial effects are focused on humans and livestock, neglecting the environment. The European Union institutions recognized this gap in the appreciation of the issue, and adopted an approach that includes to prioritize environmental tracking and to build the tools to make it economically accessible. This abstract has three main targets. Firstly, to fill the gap applying the IT methodological approach (the soil vulnerability map to antibiotic contamination) developed by De La Torre et al. (2012). Secondly, to identify the main livestock species and scenarios (agriculture and pasture) to be prioritized in surveillance efforts. Finally, to implement the code of agriculture practices and the stocking rates of grazing animals based on high vulnerability areas for antibiotic contamination. To facilitate the implementation of this risk evaluation procedure, we developed an interactive tool that allows to obtain downloadable maps of soil vulnerability to contamination for several land use (agriculture and pasture) and livestock (cattle, pig and chicken) scenarios for any veterinary antibiotics. Additionally, the tool allows to obtain a plot of the mean vulnerability
\end{abstract}


of each considered administrative unit. We implemented the European Union countries as an example, but the tool could be applied to individual countries or even regional or subnational scales.

\section{Keywords}

Antimicrobial, environmental impact assessment, agriculture, pasture, surveillance.

\section{Presenting author}

Antonio Rodríguez

\section{Presented at}

One Health EJP Annual Scientific Meeting Satellite Workshop 2021 Software Fair

\section{Acknowledgements}

The authors would like to thank the office of the Sub-Directorate General for Animal Health and Livestock Hygiene, Spanish Ministry of Agriculture, Fisheries, and Food, for their assistance in the data curation process.

\section{Funding program}

RTI208_095586_B_C21 (Spanish Ministry of Science and Innovation)

773830 (European Union's Horizon 2020 Research and Innovation program)

\section{Grant title}

Toward the availability and use of "safe, effective and affordable" antibiotics (RTI2018_095586_B_C21).

One Health European Joint Programme (RTI2018_095586_B_C21).

\section{Hosting institution}

National Institute for Agricultural and Food Research and Technology (INIA) 


\section{Author contributions}

Ana de la Torre: Conceptualization, Methodology, Supervision, Writing - Original Draft, Writing - Review \& Editing.

Antonio Rodriguez: Methodology, Resources (computing), Formal analysis, Writing Original Draft, Writing - Review \& Editing.

\section{Conflicts of interest}

The authors have no conflict of interest to declare.

\section{References}

- De La Torre A, Iglesias I, Carballo M, Ramírez P, Muñoz MJ (2012) An approach for mapping the vulnerability of European Union soils to antibiotic contamination, Sci. Science of the Total Envirnonment 414: 672-679. https://doi.org/10.1016/j.scitotenv. 2011.10.032 